\title{
Slouch Detection Using Open Pose Architecture
}

\author{
Manav Shah ${ }^{1^{*}}$, Rohit Singh ${ }^{2}$, Nimit Haria ${ }^{3}$, Krish Fadia ${ }^{4}$ and Kriti Srivastava ${ }^{5}$ \\ 1, 2, 3, 4, 5 Dwarkadas J. Sanghvi College of Engineering, Nagpur India
}

\begin{abstract}
Slouch detection refers to computer vision techniques that detect human figures in images in order to determine the current posture and check if a person is sitting ergonomically. This is an important subject because following poor posture habits is known to be one of the primary causes of back pain because of its adverse influence on the transverse abdominis muscle. Maintaining good posture could help improve one's health in the long run. This paper implements slouch detection using OpenPose architecture, which first finds the relative position of joints with respect to the body. This information then helps determine whether the human subject in a given image is sitting ergonomically or not. In this paper's implementation, high accuracy was achieved and slouch detection was made more feasible by eliminating the need for several sensors.
\end{abstract}

\section{KEY WORDS: SLOUCH DETECTION, POSTURE DETECTION, OPENPOSE ARCHITECTURE, MACHINE LEARNING.}

\section{INTRODUCTION}

In today's modern world, it has become easier than ever to find yourself slouching in-front of a laptop or television screen. Not positioning yourself correctly for a long period of time can take its toll on your muscles, joints, and ligaments. When your body gets used to being hunched over for hours, it can be easy to continue that same posture, even when you're not in front of a screen. Maintaining correct posture while sitting or otherwise, benefits both our mental [Peper, 2017] and physical health. Aside from the fact that good posture aids in preventing health issues, it also contributes to achieving a more comfortable working experience in workplaces.

On the flip side, improper posture has a detrimental effect on mood, thus leading to reduced quality of performance and satisfaction. Furthermore, various musculoskeletal

\section{ARTICLE INFORMATION}

*Corresponding Author:ma9shah@gmail.com

Received 19th Oct 2020 Accepted after revision 25th Dec 2020

Print ISSN: 0974-6455 Online ISSN: 2321-4007 CODEN: BBRCBA

Thomson Reuters ISI Web of Science Clarivate Analytics USA and Crossref Indexed Journal

\section{Clarivate
Analytics}

NAAS Journal Score 2020 (4.31)

A Society of Science and Nature Publication,

Bhopal India 2020. All rights reserved.

Online Contents Available at: http//www.bbrc.in/

Doi: http://dx.doi.org/10.21786/bbrc/13.14/37 injuries, health issues such as sprains, back pain, carpal tunnel syndrome and neck pain could also be attributed to poor sitting positions. As per [Lindsay Olson, 2013], $86 \%$ of Americans remain seated for the majority of their work-day. Hence, it is essential that people in the workplace be cognizant of their sitting posture in order to reduce health risks over a long period of time. According to [Alyazyah Alsuwaidi, 2017], 76\% admitted that their posture was not good. More than 90\% of these people were found to be experiencing back pain on a regular basis. This motivated us to implement a solution that caters to a large section of the population and this paper aims to minimize these health issues by reminding the user to correct their posture when it is detected that they are slouching. By using OpenPose architecture, improper posture was detected by taking a lateral view image as input. The position of a human's spine, ear and hip were then used to classify whether the person is slouching or sitting straight.

Related Work: In [Michael Battaglia, 2015] Michael Battaglia et al list the different approaches that have been tried before it to improve poor posture practices. Lumo Back was an independent device, Nekoze was a webcam application and keeping in mind their limitations, they came up with a Microsoft Kinect based approach, which works by tracking the left shoulder, right shoulder, center shoulder and head. These measurements are then

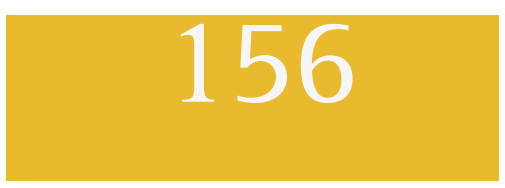


averaged out over multiple frames. They also normalize the positions such that the neck joint would lie on the $\mathrm{y}$-axis only. Therefore, the difference between positions can be found. They have used kNN for classification. The implementation is done by correctly identifying the shoulder joints and fails when the user is sitting in an extreme position (perpendicular to camera). This paper has improved upon this by considering the side view of the body.

A deep learning model to detect real time postures in gymnasts has been implemented using OpenPose model [W. Wen, 2019]. They have used the OpenPose model to extract the key points of the gymnasts. A convolutional network is used to extract features and correctly link body parts like elbows or shoulders. The method inputs an image that is processed by a VGG-19 convolutional network. Part Confidence Maps and Part Affinity Fields are then extracted by a CNN. Bipartite Matching is then used to find part association. It results in good real-time performance. This method can get key points such as ears and hips to tell the angle at which they are aligned and predict the slouching of a person. While they have implemented this model to find the posture of the gymnasts, this paper uses this model for slouch detection. Also, the OpenPose model is used for multi-person keypoint detection, but it is possible to use this for a single person detection by using only the Confidence Maps for body part detection.

In [A. Abobakr, 2017], a deep residual network is trained to calculate joint angles from a single image in order to evaluate ergonomic metrics. The primary purpose of this paper is to help develop a method to estimate the risk of work-related musculoskeletal disorders (MSDs). [Plantard P, 2017] dives into 3 assessment methods: selfreport, direct measurement and observational methods. It explains why self-report can be misleading and inaccurate, and direct methods often require high tech sensors, which make that method infeasible in several situations. This paper utilizes Kinect SDK for perceiving 3D joint positions. ResNets (deep residual convolutional networks) are heavily employed in this method. Motion capture data is mapped onto virtual human figures by using various anthropometric measures. However, this approach has trouble dealing with cluttered environments and is computationally expensive. This paper motivated us to use the joint based approach for detecting posture and to try and come up with a method that does not require dedicated gadgets.

In [Alyazyah Alsuwaidi, 2017], sensors attached to clothes are used to identify the angle of the spine. However, excessive calibration for most postures is a necessity. Accelerometers as well as gyroscopes are utilized for dynamically assessing changes in position. Even though the thoracic angle was accurately obtained, differentiating between regular bending and improper posture was problematic. Visual analysis as well as principal component analysis was carried out, but because the intended results were not met, it was concluded that a single sensor might not be reliable for this purpose.
Boundary extraction and contour selection is utilized in [8] for detecting objects. Orientation of a posture is detected by the help of analyzing head positions. Once a human figure is found in the input image, a neural network is used to find the lower or upper body after which the head is found and final posture is classified. An image is often split in order to find contours. In this paper, they create two separate and compare halves of the upper and lower body to predict the action being carried out in a video that was enhanced using filters. An accuracy of close to $90 \%$ was reached by combining head detection with lower/upper body recognition.

\section{MATERIAL AND METHODS}

This paper uses the OpenPose model [Zhe Cao, 2018], to identify the location of important joints on the human body. Below is the architecture of an OpenPose Model:

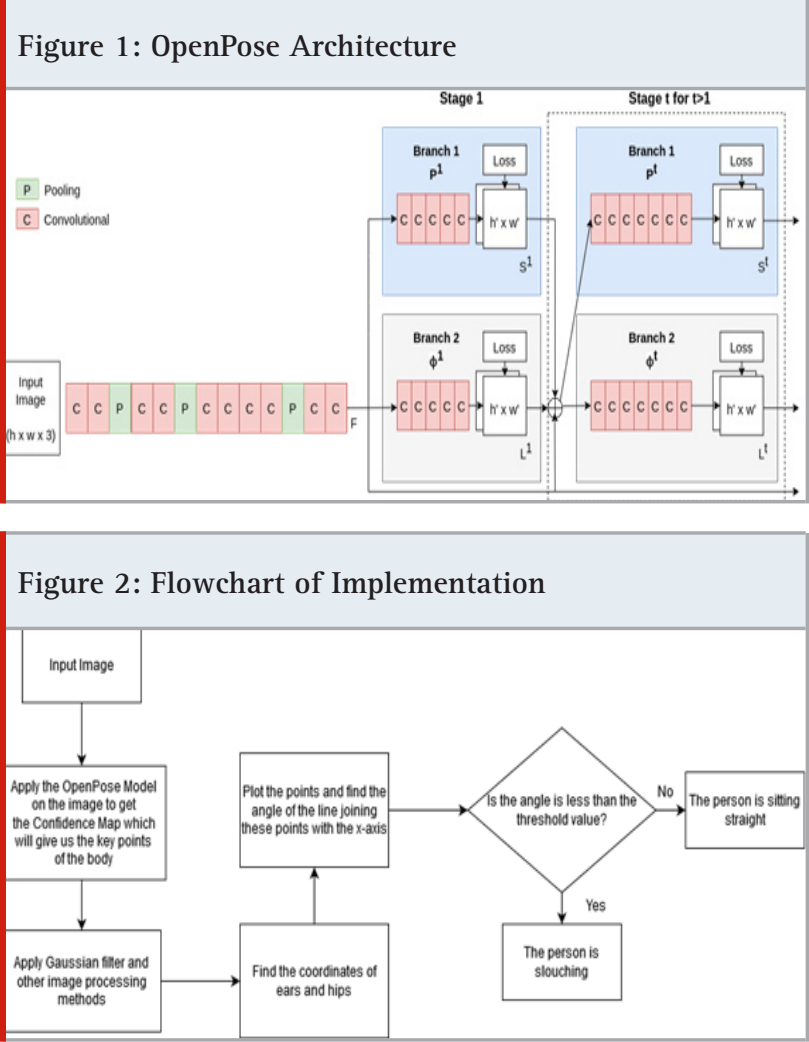

This model takes as input, an image of size ( $\mathrm{h} \mathrm{x} \mathrm{w}$ ) which is then passed through the following structure made up of two stages:

1. Stage 0: Feature map is created in this stage by the leading ten layers of the VGGNet [M. F. Haque, 2019].

2. Stage 1: This stage consists of two branches of CNN. The first branch predicts 2D Confidence Maps (S). A Confidence Map is a grayscale image with higher values at locations where a certain joint is likely to be present. For the 18 point model, the first 19 matrices give Confidence Maps. 2D vector fields (L) of Part Affinities (PAF) are determined by the second branch. The output is the confidence map of key-point pairs along with Part Affinity 
Heatmaps. OpenPose model has been trained on Coco dataset, an open-source data set with 14,000 relevant images.

In order to detect the key points, the model first finds the confidence map to detect the body part and part affinity field which would help us pair the associated body parts. Then comes the post-processing stage, where a Gaussian filter is applied. Next, the coordinates of ears and hips are calculated which are further used to find the angle and check if it is less than the threshold value.

Experimentation was performed with various angles in order to find the optimum angle for detecting slouch. Tests for the following angles were conducted: 70, 75, 80 and 85. As evident in fig. 4, accuracy rises till 80 degrees and then falls. Since 80 degrees is the peak, it was concluded that maximum accuracy is found at an angle of 80 degrees. This maximum accuracy is $95.31 \%$.

Figure 3: Threshold Angle vs Accuracy

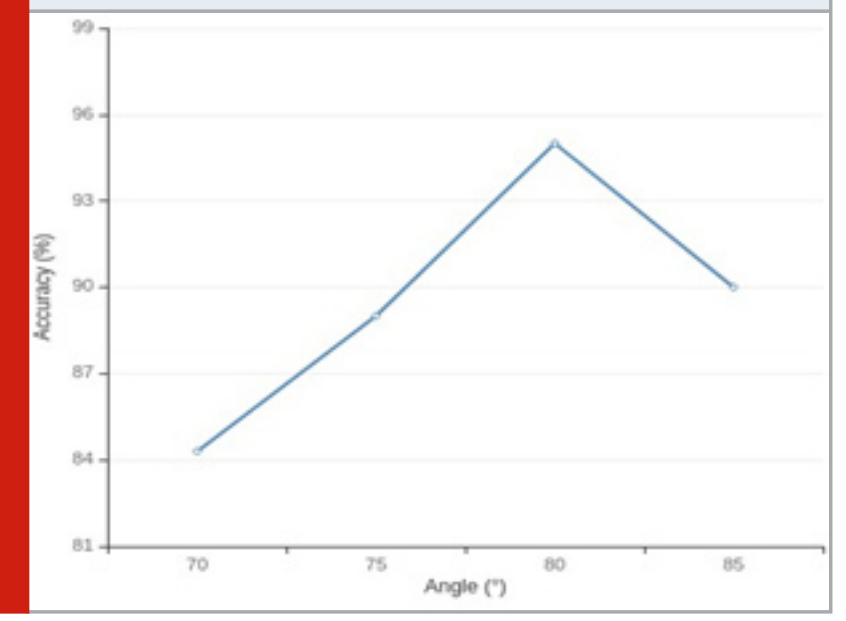

RESULTS

Accuracy, Recall, Precision and F-Score for our implementation are all shown in table 1

Table 1. Evaluation Metrics

\begin{tabular}{|l|c|}
\hline Accuracy & 0.9531 \\
\hline Recall & 0.9697 \\
\hline Precision & 0.9411 \\
\hline
\end{tabular}

Consider the following example for testing the output given by our program. Two images were provided to the model as input, in order to predict whether the person in the image is slouching or not. In the first image, the person was sitting in a straight position and in the second image, the person's back was slouched. The model correctly predicted the output as "Straight" and "Hunchback" respectively for the two images, as shown below. Furthermore, the joints that the OpenPose model returns are depicted as dots of various colours.

Figure 4: Human sitting straight

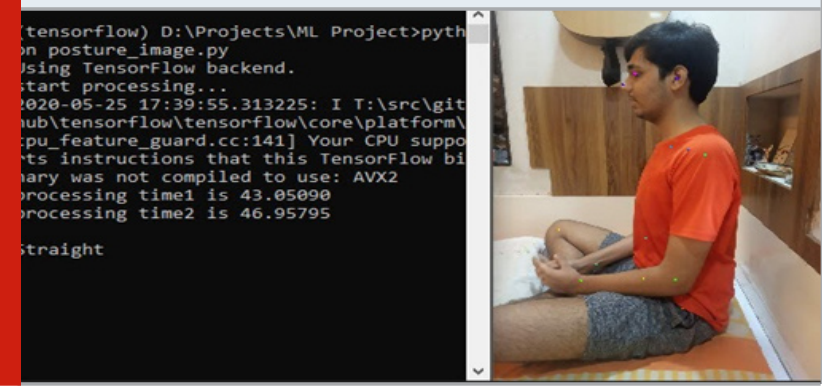

Figure 5: Human sitting with a hunchback
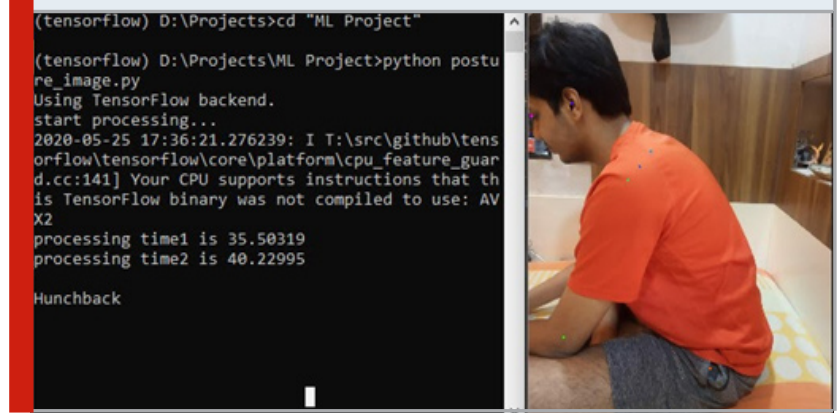

\section{CONCLUSION}

In conclusion, after considering traditional methods and their drawbacks, this paper has implemented a new approach to detect slouch in the sitting posture of humans based on OpenPose architecture. This method is able to determine whether a person in an image slouches or not with high accuracy. This paper has made an effort to make this implementation feasible by reducing the need for specialized sensors as well as improve upon most shortcomings that the earlier implementations had.

\section{REFERENCES}

A. Abobakr, D. Nahavandi, J. Iskander, M. Hossny, S. Nahavandi and M. Smets, "A kinect-based workplace postural analysis system using deep residual networks," 2017 IEEE International Systems Engineering Symposium (ISSE), Vienna, 2017, pp. 1-6, doi: 10.1109/ SysEng.2017.8088272.

A. Jalal, A. Nadeem and S. Bobasu, "Human Body Parts Estimation and Detection for Physical Sports Movements," 2019 2nd International Conference on Communication, Computing and Digital Systems (C-CODE), Islamabad, Pakistan, 2019, pp. 104-109, doi: 10.1109/C-CODE.2019.8680993

Alyazyah Alsuwaidi, Aisha Alzarouni, Dana Bazazeh, "Wearable Posture Monitoring System with vibrational feedback" in 2017 (accessed in September 2020): https:// arxiv.org/ftp/arxiv/papers/1810/1810.00189.pdf M. F. Haque, H. Lim and D. Kang, "Object Detection 
Based on VGG with ResNet Network,” 2019 International Conference on Electronics, Information, and Communication (ICEIC), Auckland, New Zealand, 2019, pp. 1-3, doi: 10.23919/ELINFOCOM.2019.8706476.

Manu Bali, Devendran V, "Human Body Posture Recognition Using Artificial Neural Networks” International Journal of Innovative Technology and Exploring Engineering (IJITEE) ISSN: 2278-3075, Volume-8, Issue-6S3, April 2019

Michael Battaglia, Maria Janezak and Brandon Slaght, "Sitting Posture Detection and Correction Using the Microsoft Kinect” in 2015: http://brandonslaght.me/ res/kinnect-posture-tracking-research-paper.pdf Peper, Erik \& Lin, I-Mei \& Harvey, Richard. (2017). SPECIAL ISSUE Posture and Mood: Implications and Applications to Therapy. Biofeedback. 45. 42-48. 10.5298/1081-5937-45.2.03.

Plantard P, Shum HPH, Le Pierres AS, Multon F,
"Validation of an ergonomic assessment method using Kinect data in real workplace conditions", Appl Ergon. 2017;65:562-569. doi:10.1016/j.apergo.2016.10.015 The Sitting Disease: Slow, Silent and Sedentary Killer (August 2013) by Lindsay Olson (accessed in August 2020): https://money.usnews.com/money/blogs/ outside-voices-careers/2013/08/22/are-you-sufferingfrom-sitting-disease

W. Wen, Y. Yang, J. Du, L. Liu and J. Wang, "Gymnastic Posture Detection Based on Deep Learning," 2019 IEEE 3rd Advanced Information Management, Communicates, Electronic and Automation Control Conference (IMCEC), Chongqing, China, 2019, pp. 1277-1281, doi: 10.1109/ IMCEC46724.2019.8983972.

Zhe Cao, Gines Hidalgo, Tomas Simon, Shih-En Wei and Yaser Sheikh, "OpenPose: Realtime Multi-Person 2D Pose Estimation using Part Affinity Fields", arXiv:1812.0808 